\section{Commentary: Cell therapy for spinal regeneration - implications for recovery after complex aortic surgery}

Vishnu Vasanthan, MD, Ali Fatehi Hassanabad, MD, and Paul W. M. Fedak, MD, PhD, FRCSC

Spinal ischemia-reperfusion injury is a dreaded complication affecting some patients after complex aortic surgery, causing paraplegia and loss of quality of life. ${ }^{1}$ Contemporary neuroprotection strategies will optimize spinal cord perfusion, pressures, and temperature but do not address postischemic injury by enhancing neurorepair and regeneration. ${ }^{2}$ Cellular therapy for spinal regeneration may provide a unique approach to preserve postoperative quality of life.

In this issue of JTCVS Open, Nakai and colleagues ${ }^{3}$ investigate the therapeutic potential of human bone marrow mesenchymal stromal cells (hBM-MSCs) in spinal ischemia-reperfusion injury in a novel murine model. Spinal ischemia-reperfusion injury was induced by clamping both the aortic arch distal to the left carotid and the proximal left subclavian artery for 5 minutes. Intravenous administration of hBM-MSCs was performed 2 hours after reperfusion. Histology showed localization of hBM-MSCs in the spinal cord, lung, spleen, and kidney. Motor functional recovery was enhanced in the cellular treatment group, accompanied by improved lumbar spinal cord motor neuron density. Reverse transcription polymerase chain reaction results also showed a transcriptional shift in the spinal cord favoring anti-inflammatory and angiogenic pathways. Overall, the authors highlight the potential reparative capacity of hBM-MSC therapy after spinal cord ischemia.

\footnotetext{
From the Section of Cardiac Surgery, Department of Cardiac Sciences, Libin Cardiovascular Institute, Cumming School of Medicine, University of Calgary, Calgary, Alberta, Canada.

Disclosures: The authors reported no conflicts of interest.

The Journal policy requires editors and reviewers to disclose conflicts of interest and to decline handling or reviewing manuscripts for which they may have a conflict of interest. The editors and reviewers of this article have no conflicts of interest.

Received for publication June 15, 2021; revisions received June 15, 2021; accepted for publication June 18, 2021; available ahead of print July 5, 2021.

Address for reprints: Paul W. M. Fedak, MD, PhD, FRCSC, C849, 1403 29th St NW, Calgary, Alberta T2N 2T9, Canada (E-mail: paul.fedak@gmail.com).

JTCVS Open 2021;7:45-6

2666-2736

Copyright (C) 2021 The Author(s). Published by Elsevier Inc. on behalf of The American Association for Thoracic Surgery. This is an open access article under the CC BY-NC-ND license (http://creativecommons.org/licenses/by-nc-nd/4.0/).

https://doi.org/10.1016/j.xjon.2021.06.016
}

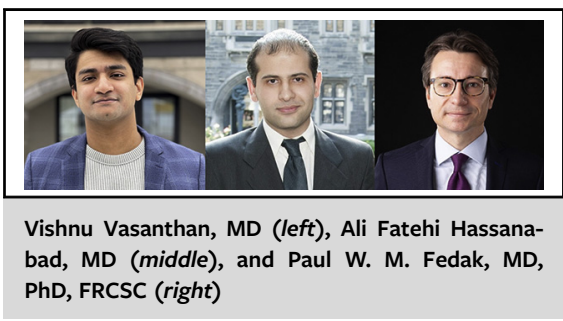

CENTRAL MESSAGE

Cell-based regenerative strategies may provide unique avenues for postoperative repair of ischemic spinal cord injuries and preserve quality of life after complex aortic interventions.

This study provides the first step toward the use of novel, cell-based treatments to address spinal ischemia-reperfusion injury after complex aortic surgery. While the data are exciting, there are important limitations. Systemic delivery of cells may limit regional engraftment at the site of interest and decrease effectiveness. Cells homing to other organs off-target could have serious side effects. The underlying cell and molecular mechanisms mediating the observed functional benefits are also unclear. Understanding whether hBM-MSCs require direct contact with the spinal cord, if they differentiate into key cell populations, or if paracrine release of reparative biomolecules is key for further development. Nonetheless, the data demonstrate the value of exploring this novel cellular therapy.

The future of ischemic spinal cord repair and regeneration is promising. Targeted administration by direct injection, intrathecal delivery, or by arterial fluoroscopic catheter approaches may be capable of delivering cell therapies without sequestration outside the target organ. Understanding mechanisms for postischemic spinal cord repair, such as critical paracrine mediators, may also facilitate future acellular therapies. Acellular repair can mitigate key barriers of stem cell therapy, such as donor-cell availability, engraftment variability, and numerous regulatory challenges. ${ }^{4,5}$ Biomaterials from extracellular matrix or synthetic origins have been shown to provide bioactive factors that upregulate endogenous mechanisms of repair or act as a base to improve cell engraftment. ${ }^{6-9}$ Further exploring optimal administration strategies and better defining reparative mechanisms may facilitate targeted patient-specific strategies that will protect and enhance the quality of life of patients undergoing complex aortic surgery. 


\section{References}

1. Svensson LG, Crawford ES, Hess KR, Coselli JS, Safi HJ. Experience with 1509 patients undergoing thoracoabdominal aortic operations. J Vasc Surg. 1993;17: 357-70.

2. Erbel R, Aboyans V, Boileau C, Bossone E, Bartolomeo RD, Eggebrecht H, et al. 2014 ESC guidelines on the diagnosis and treatment of aortic diseases: document covering acute and chronic aortic diseases of the thoracic and abdominal aorta of the adult. The task force for the diagnosis and treatment of aortic diseases of the European Society of Cardiology (ESC). Eur Heart J. 2014;35: 2873-926.

3. Nakai H, Fujita Y, Masuda S, Komatsu M, Tani A, Okita Y, et al. Intravenous injection of adult human bone marrow mesenchymal stromal cells attenuates spinal cord ischemia/reperfusion injury in a murine aortic arch crossclamping model. $J$ Thorac Cardiovasc Surg Open. 2021;7:23-40.

4. Trounson A, McDonald C. Stem cell therapies in clinical trials: progress and challenges. Cell Stem Cell. 2015;17:11-22.
5. Kleiderman E, Boily A, Hasilo C, Knoppers BM. Overcoming barriers to facilitate the regulation of multi-centre regenerative medicine clinical trials. Stem Cell Res Ther. 2018;9:307.

6. Mewhort HEM, Svystonyuk DA, Turnbull JD, Teng G, Belke DD, Guzzardi DG, et al. Bioactive extracellular matrix scaffold promotes adaptive cardiac remodeling and repair. JACC Basic Transl Sci. 2017;2:450-64.

7. Svystonyuk DA, Mewhort HEM, Hassanabad AF, Heydari B, Mikami Y, Turnbull JD, et al. Acellular bioscaffolds redirect cardiac fibroblasts and promote functional tissue repair in rodents and humans with myocardial injury. Sci Rep. 2020;10:9459.

8. Yang C-Y, Song B, Ao Y, Nowak AP, Abelowitz RB, Korsak RA, et al. Biocompatibility of amphiphilic diblock copolypeptide hydrogels in the central nervous system. Biomaterials. 2009;30:2881-98.

9. Katoh H, Yokota K, Fehlings MG. Regeneration of spinal cord connectivity through stem cell transplantation and biomaterial scaffolds. Front Cell Neurosci. 2019;13:248. 